Rev. Bras. Saúde Prod. Anim., Salvador, v.16, n.2, p.278-289 abr./jun.., 2015 http://www.rbspa.ufba.br

\title{
Influência de fatores ambientais sobre pesos pré-desmama de bovinos cruzados Aberdeen Angus x Nelore
}

\author{
Influence of environmental factors on pre-weaning weights of crossed cattle Aberdeen \\ Angus $x$ Nelore
}

\author{
SILVA, Josineudson Augusto II de Vasconcelos ${ }^{1 *}$; RIBEIRO, Cinthia Brosco ${ }^{1}$; \\ MAIORANO, Amanda Marchi ${ }^{2}$; HADLICH, Janaina Conte ${ }^{1}$; CURI, Rogerio \\ Abdallah ${ }^{1}$; OLIVEIRA, Henrique Nunes ${ }^{2}$; LAMARE, Maria ${ }^{3}$; MEIRELLES, Paulo \\ Roberto de Lima ${ }^{1}$
}

\footnotetext{
${ }^{1}$ Universidade Estadual Paulista "Júlio de Mesquita Filho", Faculdade de Medicina Veterinária e Zootecnia, Departamento de Melhoramento e Nutrição Animal, Botucatu, São Paulo, Brasil.

${ }^{2}$ Universidade Estadual Paulista "Júlio de Mesquita Filho", Faculdade de Ciências Agrárias e Veterinárias, Departamento de Zootecnia, Jaboticabal, São Paulo, Brasil.

${ }^{3}$ Fazenda Sant'Anna, Rancharia, São Paulo, Brasil.

*Endereço eletrônico: jaugusto@fmvz.unesp.br
}

\section{RESUMO}

O objetivo com este trabalho foi estimar efeitos de ambiente (idade da vaca ao parto, data juliana de nascimento e idade do bezerro à desmama) sobre os pesos ajustados aos 120 (P120) e 205 (P205) dias de idade em bovinos mestiços Angus x Nelore. O banco de dados foi formado com informações de 11.271 animais, nascidos no período de 1987 a 2004. Os animais eram filhos de matrizes com proporções de heterozigoses iguais a 0,$0 ; 0,375 ; 0,4688 ; 0,5$; 0,75 e 1,0. Os pesos, P120 e P210, foram padronizados com base no ganho diário de peso por meio da interpolação da pesagem próxima a idade a ser padronizada e do peso mensurado anteriormente. Para obtenção dos componentes de (co)variância e parâmetros genéticos das características, análises sob inferência bayesiana foram realizadas com três modelos estatísticos, diferindo quanto aos efeitos ambientais. Os valores genéticos foram obtidos para os três modelos e foram comparados por correlações de ordem Spearman e pela porcentagem de touros selecionados em comum. Os valores das estimativas de herdabilidade a posteriori obtidos no melhor modelo foi 0,20 para ambas as características (P120 e P205). O estudo dos valores genéticos mostrou alterações na classificação dos animais de acordo com os modelos. A inclusão de classe de data juliana ao nascimento, classe de idade da mensuração e interação da classe de idade da mãe ao parto e da heterozigose materna no momento da escolha dos reprodutores foi importante de modo a permitir maior precisão na avaliação genética e esquemas de seleção mais acurados.

Palavras-chave: características de crescimento, herdabilidade, inferência bayesiana

\section{SUMMARY}

The aim of this study was to estimate the environmental effects (calving age, julian date of birth and weaning age) on the adjusted weights to 120 (P120) and 205 (P205) days of crossbred cattle Angus x Nelore. The database was formed with information from 11,271 animals, borned from 1987 to 2004. Maternal heterozygosity proportions were equal to 0.0 , $0.375,0.4688,0.5,0.75$ and 1.0 . The weights, P120 and P210, were standardized based on the weight daily gain by interpolation of the weighing close to the standardized age and weight measured previously. The (co) variance components and genetic parameters were obtained by Bayesian inference analyzes performed with three statistical models, differing as to the environmental effects. Breeding values were obtained for three models and were compared by Spearman correlations and by percentage of selected bulls in common. 
Heritability estimates values obtained in the best model was 0.20 for both traits (P120 and P205). Study of genetic values showed changes in the classification of animals according to models. Inclusion of julian date class of birth, age class of the animal in the measurement and interaction of calving age class and maternal heterozygosity in the selection of parents was important to allow greater precision in genetic evaluation of herd.

Keywords: Bayesian inference, growth traits, heritability

\section{INTRODUÇÃO}

A pecuária de corte no Brasil está cada vez mais competitiva, tornando necessários aumentos constantes de produtividade. Para isto são utilizados métodos de melhoramento animal como o cruzamento industrial, para o qual existem várias publicações atestando $\mathrm{o}$ seu benefício no Brasil, principalmente nas características de crescimento.

Para se obter maior acurácia na seleção de animais geneticamente superiores, é importante a identificação e a quantificação de fatores ambientais que atuem sobre as características, os quais devem ser considerados e terem seus efeitos eliminados, utilizando-se fatores de ajuste ou outras formas de correção.

A expressão das características em determinado indivíduo é influenciada por efeitos genéticos, ambientais $\mathrm{e}$ interação entre genótipo e ambiente. Um dos fatores ambientais que apresenta forte influência sobre as características de crescimento dos bezerros é a idade da vaca ao parto, influenciando à produção de leite e ao comportamento materno filial. Dessa forma, identificar os efeitos de ambiente atuantes sobre as características $\mathrm{e}$ possíveis interações tem importância, pois possibilita identificação dos indivíduos que possuem maior expressão das características fenotípicas.

Segundo Bocchi \& Albuquerque (2005) o ganho médio diário do período prédesmame e o peso ao desmame são características que podem ser préajustadas para os fatores de meio, tais como a idade da vaca ao parto (IDV), a data juliana de nascimento (DJN) e a idade do bezerro à desmama (IDB). A DJN é a data de nascimento do bezerro, considerando o calendário juliano que varia de 1 a 365 dias.

$\mathrm{O}$ estudo de caracteres relacionados ao período pré-desmama são de fundamental importância econômica em bovinos de corte, uma vez que podem auxiliar na avaliação da habilidade materna da vaca, além do potencial genético de crescimento dos bezerros (PEREIRA \& MUNIZ, 2013).

Nesse sentido, a identificação e quantificação de efeitos ambientais que atuem sobre características desta natureza são relevantes para programas de melhoramento, uma vez que podem facilitar a identificação do potencial genético dos animais e, consequentemente, esquemas de seleção mais acurados.

Assim, o objetivo deste trabalho foi estimar efeitos de ambiente (idade da vaca ao parto, data juliana de nascimento e idade do bezerro à desmama) sobre os pesos ajustados aos 120 e 205 dias de idade em bovinos mestiços Angus X Nelore, a fim de verificar a importância destes efeitos na seleção de reprodutores.

\section{MATERIAL E MÉTODOS}

A população de bovinos considerada neste trabalho tem por base animais com diferentes composições genéticas das raças Aberdeen Angus e Nelore, 
filhos de matrizes com heterozigoses esperadas iguais a 0,$0 ; 0,375 ; 0,4688 ; 0,5$; 0,75 e 1,0 . A produção das progênies foi direcionada na estruturação dos rebanhos para obtenção de animais Brangus, com objetivo de registrá-los na Associação Brasileira de Brangus.

O programa de melhoramento genético foi iniciado em 1995 com duas propriedades e, com o passar dos anos, foram agregados novos rebanhos. No ano de 2004, doze fazendas participavam do programa. O programa tem projeto de execução delineado e foi credenciado em 1995 no Ministério da Agricultura Pecuária e Abastecimento. $\mathrm{O}$ credenciamento permitiu a emissão de Certificado Especial de Identificação e Produção (CEIP) para os animais (machos e fêmeas) que apresentassem superioridade genética em comparação com seus contemporâneos. As características utilizadas para emissão do CEIP eram o peso à desmama, peso aos 550 dias de idade, musculosidade e perímetro escrotal, constantes também do índice de seleção do programa.

As características zootécnicas consideradas na avaliação genética foram mensuradas, nas propriedades, por equipe previamente treinada. Os dados coletados foram enviados ao Departamento de Melhoramento e Nutrição Animal da Faculdade de Medicina Veterinária e Zootecnia, Unesp, Botucatu, onde realizou-se sua consistência.

A base de dados completa incluía informações das características (ponderais, reprodutivas ou de avaliação visual) utilizadas como critérios de seleção pelo programa, bem como informações de genealogia. No presente trabalho, foram utilizados os pesos aos 120 (P120) e 205 (P205) dias de idade padronizados com base no ganho diário de peso por meio da interpolação da pesagem próxima a idade a ser padronizada e do peso mensurado anteriormente. As pesagens utilizadas para este fim foram obtidas em idades que variaram 90 dias a mais ou menos das idades consideradas.

A análise foi realizada com a utilização de banco de dados contendo 11.271 animais, nascidos no período de 1987 a 2004, filhos de 449 pais e 6.693 mães. Para a formação do pedigree foi criado um "dicionário de touros", no qual se encontravam todos os pais dos touros dos rebanhos participantes do programa. Este arquivo permitiu unificar a identificação dos touros utilizados em mais de um rebanho.

Os fatores ambientais avaliados foram idade da vaca ao parto, data juliana de nascimento e idade do bezerro à desmama. Anteriormente às análises, foram formadas classes para todos os fatores ambientais considerados. $\mathrm{O}$ número de observações e as médias observadas para as características analisadas de acordo com a classe de idade da mãe ao parto, classe de data juliana de nascimento e classe de idade do bezerro à mensuração, estão apresentadas nas Tabelas 1, 2 e 3, respectivamente.

A idade da vaca ao parto variou de 2 a 15 anos, sendo dividida em seis classes $\left(1^{\mathrm{a}}\right.$ até dois anos de idade, $2^{\mathrm{a}}=$ três anos, $3^{\mathrm{a}}=$ quatro anos, $4^{\mathrm{a}}=$ maior de $4 \mathrm{e}$ menor de 10 anos, $5^{\mathrm{a}}=$ maior de $9 \mathrm{e}$ menor de 12 anos e $6^{\mathrm{a}}=12$ ou mais anos de idade).

A data juliana de nascimento variou de $1^{\circ}$ de janeiro a $365^{\circ}$ de dezembro, ou seja, havia nascimentos ao longo do ano, pois algumas fazendas não possuíam estação de monta.

A idade de desmama variou de 115 a 295 dias de idade. Foram formadas classes a cada 20 dias para idade do animal à desmama e data juliana de nascimento. Para todos os fatores ambientais, as classes que possuíam poucos animais foram consideradas nas 
Rev. Bras. Saúde Prod. Anim., Salvador, v.16, n.2, p.278-289 abr./jun.., 2015 http://www.rbspa.ufba.br ISSN 15199940

análises com intuito de verificar como afetariam os resultados e para possibilitar maior discussão.

As características foram avaliadas separadamente, por meio de quadrados mínimos, utilizando o procedimento GLM (SAS, 2010). O modelo estatístico utilizado, descrito em forma matricial, foi: $y=X \beta+e$, em que, $y$ é o vetor de dados (P120 e P205); X é a matriz de incidência associada aos efeitos sistemáticos; $\beta$ é o vetor de efeitos sistemáticos e e é o vetor de resíduos, em que $\mathrm{E}(\mathrm{y})=\mathrm{X} \beta ; \mathrm{E}(\mathrm{e})=0$ e $\operatorname{Var}(\mathrm{e})=$ I * $\sigma_{e}^{2}$, sendo que I é a matriz identidade e $\sigma^{2}$ é o componente de variância do resíduo.

Tabela 1. Número de observações (N), média (M), desvio padrão (DP) e coeficiente de variação (CV) para as características peso aos 120 (P120) e 205 (P205) dias de idade em função da classe de idade da mãe ao parto (CIMP) de bovinos cruzados Angus x Nelore

\begin{tabular}{ccccccccc}
\hline \multirow{2}{*}{ CIMP } & \multicolumn{9}{c}{ P120 } & $\mathrm{N}$ & $\mathrm{M}$ & $\mathrm{DP}$ & $\mathrm{CV}$ & $\mathrm{N}$ & $\mathrm{M}$ & $\mathrm{DP}$ & $\mathrm{CV}$ \\
\cline { 2 - 9 } & 135 & 114,8 & 25,0 & 21,8 & 451 & 179,7 & 38,7 & 21,5 \\
2 & 511 & 126,4 & 21,7 & 17,2 & 1695 & 184,1 & 34,2 & 18,6 \\
3 & 298 & 130,1 & 20,2 & 15,5 & 1448 & 186,2 & 33,9 & 18,2 \\
4 & 634 & 132,6 & 22,0 & 16,6 & 1813 & 192,4 & 35,5 & 18,4 \\
5 & 90 & 133,9 & 17,8 & 13,3 & 473 & 186,4 & 34,7 & 18,6 \\
6 & 474 & 134,2 & 22,4 & 16,7 & 1298 & 193,8 & 34,9 & 18,0 \\
\hline
\end{tabular}

Tabela 2. Número de observações (N), média (M), desvio padrão (DP) e coeficiente de variação (CV) para as características peso aos 120 (P120) e 205 (P205) dias de idade em função da classe de data juliana de nascimento (CDJN) de bovinos cruzados Angus x Nelore

\begin{tabular}{|c|c|c|c|c|c|c|c|c|c|}
\hline \multirow{2}{*}{ CDJN } & \multirow{2}{*}{$\begin{array}{l}\text { Data juliana de } \\
\text { nascimento }\end{array}$} & \multicolumn{4}{|c|}{ P120 } & \multicolumn{4}{|c|}{ P205 } \\
\hline & & $\mathrm{N}$ & M & DP & $\mathrm{CV}$ & $\mathrm{N}$ & $\mathrm{M}$ & DP & $\mathrm{CV}$ \\
\hline 1 & $1 \mathrm{jan}-20 \mathrm{jan}$ & 46 & 124,2 & 30,3 & 24,4 & 112 & 172,0 & 32,9 & 19,2 \\
\hline 2 & $21 \mathrm{jan}-9 \mathrm{fev}$ & 87 & 121,2 & 18,3 & 15,1 & 183 & 173,2 & 29,0 & 16,7 \\
\hline 3 & $10 \mathrm{fev}-1 \mathrm{mar}$ & 85 & 118,0 & 20,1 & 17,0 & 199 & 173,7 & 30,0 & 17,3 \\
\hline 4 & $2 \operatorname{mar}-21 \operatorname{mar}$ & 121 & 118,2 & 19,1 & 16,2 & 229 & 176,6 & 34,5 & 19,5 \\
\hline 5 & $22 \mathrm{mar}-10 \mathrm{abr}$ & 123 & 124,8 & 20,0 & 16,0 & 204 & 179,9 & 37,9 & 21,1 \\
\hline 6 & $11 \mathrm{abr}-30 \mathrm{abr}$ & 18 & 120,6 & 21,5 & 17,8 & 70 & 174,3 & 31,3 & 17,9 \\
\hline 7 & $1 \mathrm{mai}-20$ mai & 12 & 149,2 & 27,2 & 18,2 & 41 & 188,3 & 36,4 & 19,3 \\
\hline 8 & $21 \mathrm{mai}-10$ jun & 4 & 135,0 & 29,6 & 21,9 & 40 & 185,4 & 33,1 & 17,8 \\
\hline 9 & 11 jun -31 jun & 20 & 138,1 & 24,2 & 17,6 & 49 & 189,5 & 39,6 & 20,9 \\
\hline 10 & $1 \mathrm{jul}-20 \mathrm{jul}$ & 21 & 135,6 & 21,7 & 16,0 & 213 & 164,1 & 27,8 & 17,0 \\
\hline 11 & 21 jul - 9 ago & 35 & 121,7 & 23,2 & 19,1 & 697 & 180,2 & 30,3 & 16,8 \\
\hline 12 & 10 ago -29 ago & 106 & 131,8 & 26,2 & 19,9 & 135 & 188,4 & 31,8 & 16,9 \\
\hline 13 & 30 ago -18 set & 290 & 132,9 & 22,0 & 16,6 & 147 & 192,3 & 32,2 & 16,8 \\
\hline 14 & 19 set -8 out & 379 & 135,6 & 20,0 & 14,8 & 139 & 197,2 & 33,2 & 16,8 \\
\hline 15 & 9 out -28 out & 276 & 131,3 & 21,0 & 16,0 & 121 & 199,8 & 37,1 & 18,6 \\
\hline 16 & 29 out -17 nov & 175 & 135,6 & 24,8 & 18,3 & 820 & 197,4 & 42,5 & 21,5 \\
\hline 17 & $18 \mathrm{nov}-7 \mathrm{dez}$ & 203 & 130,6 & 21,2 & 16,2 & 556 & 180,7 & 32,3 & 17,9 \\
\hline 18 & $8 \mathrm{dez}-31 \mathrm{dez}$ & 141 & 126,8 & 20,6 & 16,3 & 314 & 174,4 & 35,9 & 20,6 \\
\hline
\end{tabular}


Rev. Bras. Saúde Prod. Anim., Salvador, v.16, n.2, p.278-289 abr./jun.., 2015 http://www.rbspa.ufba.br ISSN 15199940

Tabela 3. Número de observações (N), média (M), desvio padrão (DP) e coeficiente de variação (CV) para as características peso aos 120 (P120) e 205 (P205) dias de idade em função da classe de idade na mensuração (CID) de bovinos cruzados Angus x Nelore

\begin{tabular}{ccccccccccc}
\hline \multirow{2}{*}{ CID } & \multicolumn{9}{c}{ P120 } & \multicolumn{9}{c}{ P205 } \\
\cline { 2 - 11 } & Idade & N & M & DP & CV & Idade & N & M & DP & CV \\
\hline 1 & $31-50$ & 6 & 132,7 & 24,7 & 18,6 & $115-134$ & 173 & 230,7 & 54,3 & 23,6 \\
2 & $51-70$ & 15 & 131,1 & 40,0 & 30,5 & $135-154$ & 536 & 225,7 & 43,6 & 19,3 \\
3 & $71-90$ & 97 & 141,8 & 22,8 & 16,1 & $155-174$ & 868 & 203,8 & 32,7 & 16,1 \\
4 & $91-110$ & 226 & 125,4 & 21,5 & 17,2 & $175-194$ & 1583 & 197,3 & 31,0 & 15,7 \\
5 & $111-130$ & 259 & 130,9 & 27,1 & 20,7 & $195-214$ & 2046 & 189,6 & 30,4 & 16,0 \\
6 & $131-150$ & 241 & 133,3 & 22,2 & 16,7 & $215-234$ & 2072 & 181,6 & 30,2 & 16,6 \\
7 & $151-170$ & 271 & 132,2 & 21,2 & 16,1 & $235-254$ & 1378 & 172,0 & 28,3 & 16,5 \\
8 & $171-190$ & 422 & 133,1 & 21,5 & 16,2 & $255-274$ & 367 & 163,7 & 26,7 & 16,3 \\
9 & $191-210$ & 605 & 125,2 & 19,3 & 15,4 & $275-295$ & 155 & 158,0 & 24,6 & 15,6 \\
\hline
\end{tabular}

Para ambas as características estudadas, P120 e P205, os efeitos sistemáticos apreciados foram grupo de contemporâneo (GC), classe de idade na mensuração das características (CID), covariável linear da idade na mensuração da característica (IDM), classe de data juliana de nascimento (CDJN), classe de idade da mãe ao parto (CIMP), heterozigose individual (HTI), heterozigose materna (HTM) e interação entre classe de idade da mãe ao parto e heterozigose materna (CIMP * HTM). O GC foi composto por fazenda, ano e trimestre de nascimento, sexo e grupo de manejo nas respectivas idades consideradas das características.

Análises utilizando inferência bayesiana, por meio do programa GIBBS2F90 (MISZTAL et al., 2002), foram realizadas para obtenção dos componentes de (co)variância e parâmetros genéticos das características P120 e P205, com uso de três modelos descritos a seguir:

- Modelo 1: Efeitos sistemáticos de GC, classe de data juliana, classe de idade na mensuração, heterozigose individual e interação da classe de idade da mãe ao parto e da heterozigose materna, mais efeitos genéticos direto, materno e ambiente permanente.

- Modelo 2: Efeitos sistemáticos de GC, classe de data juliana, classe de idade da mãe ao parto, heterozigose materna e heterozigose individual, covariável linear da idade na mensuração, mais efeitos genéticos direto, materno $\mathrm{e}$ ambiente permanente.

- Modelo 3: Efeitos sistemáticos de GC, interação da classe de idade da mãe ao parto e da heterozigose materna, e heterozigose individual, covariável linear da idade na mensuração, mais efeitos genéticos direto, materno $\mathrm{e}$ ambiente permanente.

Para todas as análises foram obtidos 500.000 ciclos, com período de descarte e intervalo amostral de 20.000 e 20 ciclos, respectivamente. Análises de convergência e descrição dos resultados pós amostragem de Gibbs foram realizadas com o programa POSTGIBBSF90, desenvolvido por S. Tsuruta (MISZTAL et al., 2002).

$\mathrm{O}$ deviance information criterion (DIC) foi utilizado como critério de comparação entre os modelos para determinar qual modelo apresentou melhor ajuste, sendo que $\theta$ representa os 
Rev. Bras. Saúde Prod. Anim., Salvador, v.16, n.2, p.278-289 abr./jun.., 2015 http://www.rbspa.ufba.br

parâmetros do modelo, então o DIC foi calculado como:

$\mathrm{DIC}=\overline{\mathrm{D}}(\theta)+\mathrm{P}_{\mathrm{D}(\mathrm{\theta})}$

em que, $\bar{D}(\theta)$ é a média posterior do desvio que é $-2 \log p(y \mid \theta)$. O número efetivo de parâmetros $\left(\mathrm{P}_{\mathrm{D}(\mathbf{G})}\right)$ é determinado pela fórmula:

$\mathrm{P}_{\mathrm{D}(\bar{\theta})}=\overline{\mathrm{D}}(\theta)-\mathrm{D}(\bar{\theta})$, onde $\bar{\theta}$ é a média posterior de $\theta$. Baixos valores de DIC indicam melhor ajuste, com penalização para modelos complexos com base no número efetivo de parâmetros (SPIEGELHALTER et al., 2002). Os mesmos autores sugerem que a subtração do DIC de diferentes modelos, resultando em valor igual a 7 , representa importante diferença de ajuste dos modelos.

O DIC e o menor valor de variância residual foram utilizados para definir qual modelo foi melhor em estimar os componentes genéticos. Os valores genéticos foram obtidos utilizando, nos três modelos descritos, os componentes genéticos gerados pelo melhor modelo e a mesma base de dados. Os resultados dos modelos foram comparados de duas formas: por meio das correlações de ordem Spearman entre os valores genéticos do conjunto de animais constante no pedigree e do arquivo com somente os touros, comparando as diferenças na classificação dos animais por modelos; e por meio da porcentagem de touros selecionados em comum com as diferentes abordagens, sendo a porcentagem inspecionada em dois percentuais de animais selecionados.

\section{RESULTADOS E DISCUSSÃO}

As médias observadas das características P120 e P205 foram 130,1 e $189,1 \mathrm{~kg}$, respectivamente. Na Tabela 4, são apresentados os resultados das análises de variância para as características avaliadas. Os efeitos considerados na modelagem foram significativos $(p<0,01)$, com exceção da CDJN e da HTI para P120.

Tabela 4. Fontes de variação, graus de liberdade (GL) e quadrados médios (QM) para as características peso aos 120 (P120) e 205 (P205) dias de idade em bovinos cruzados Angus X Nelore

\begin{tabular}{lccccc}
\hline \multirow{2}{*}{ Fontes de variação } & \multicolumn{2}{c}{ P120 } & & \multicolumn{2}{c}{ P205 } \\
\cline { 2 - 3 } & GL & QM & & GL & QM \\
\cline { 2 - 3 } \cline { 5 - 6 } CDJN & 132 & $2273,1^{* *}$ & & 292 & $9990,8^{* *}$ \\
CID & 17 & 403,4 & & 17 & $2750,8^{* *}$ \\
HTI & 8 & $2261,5^{* *}$ & & 8 & $27996,9^{* *}$ \\
CIMP * HTM & 6 & 223,7 & & 6 & $2866,5^{* *}$ \\
Resíduo & 34 & $1263,2^{* *}$ & & 34 & $8434,9^{* *}$ \\
$\mathrm{R}^{2}$ & 1944 & 276,6 & & 8820 & 596,4 \\
\hline
\end{tabular}

$\mathrm{GC}=$ grupo de contemporâneo; CDJN = classe de data juliana de nascimento; CID = classe de idade na mensuração das características P120 e P205; HTI = heterozigose individual; CIMP * HTM = interação entre classe de idade da mãe ao parto e heterozigose materna; $\mathrm{R}^{2}=$ coeficiente de determinação.

**As estatísticas foram significativas ao nível de significância de $1 \%(\mathrm{p}<0,01)$. 
As soluções dos quadrados mínimos ajustadas pelo modelo de acordo com a CDJN, CID e a interação CIMP x HTM são apresentadas nas Figuras 1, 2 e 3.

$\mathrm{O}$ efeito da data juliana de nascimento no peso aos 205 foi significativo $(\mathrm{p}<0,01)$ e pode ser verificado que, a partir da classe 10 ( 1 jul - 20 jul), os pesos apresentam menores valores. Isto também foi verificado no trabalho de Jorge Junior et al. (2001), que separaram as classes da mesma forma, mas somente a partir da classe 13 os pesos apresentaram-se menores. Deve ser observado que os animais com data juliana menor são os animais mais velhos e, portanto, mais pesados na data de desmama.
No caso do P120, em que o efeito de CDJN não foi significativo ( $p>0,05)$, os resultados possivelmente demonstram que, independente da época de nascimento, os animais conseguem obter pesos similares nestas idades, e sugerem que $o$ efeito materno complementa o peso e tem grande importância nesta idade, permitindo ganho de peso similar aos animais de outras idades. Houveram variações nos valores médios das soluções dos quadrados mínimos para a característica P120 devido a CDJN (Figura 1), contudo não foi verificada diferença estatística significativa $(p>0,05)$ para este efeito.
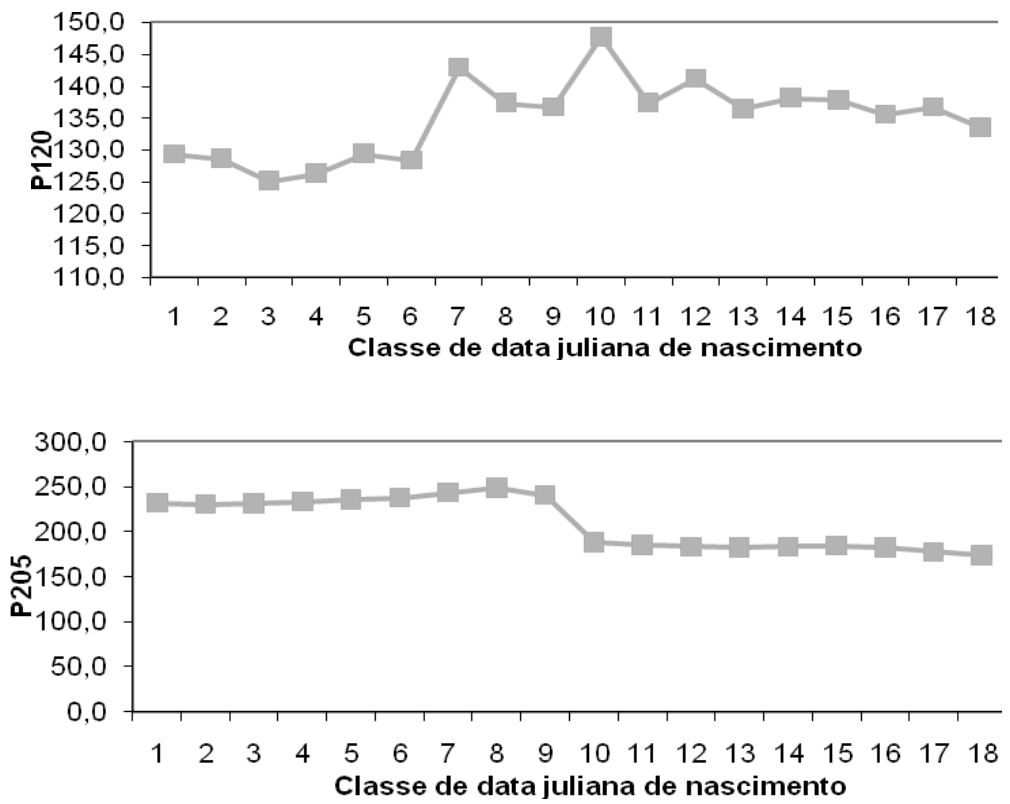

Figura 1. Médias ajustadas das características peso aos 120 (P120) e 205 (P205) dias de idade de acordo com a classe de data juliana de nascimento (CDJN) de bovinos cruzados Angus X Nelore

A classe de idade apresentou efeito significativo $(\mathrm{p}<0,01)$ para $\mathrm{P} 120 \mathrm{e}$ P205. Na padronização do peso foi considerado o ganho médio diário, o qual será menor no caso do animal possuir maior idade. Sendo assim, conforme a classe de idade aumenta, ocorre diminuição dos pesos 
Rev. Bras. Saúde Prod. Anim., Salvador, v.16, n.2, p.278-289 abr./jun.., 2015 http://www.rbspa.ufba.br ISSN 15199940

padronizados, indicando possivelmente menores ganhos de peso nos animais mais velhos (Figura 2). Este fato também foi verificado no trabalho de
Paz (1999) para ganho de peso do nascimento à desmama, o qual diminuiua medida que a idade ao desmame aumentou.
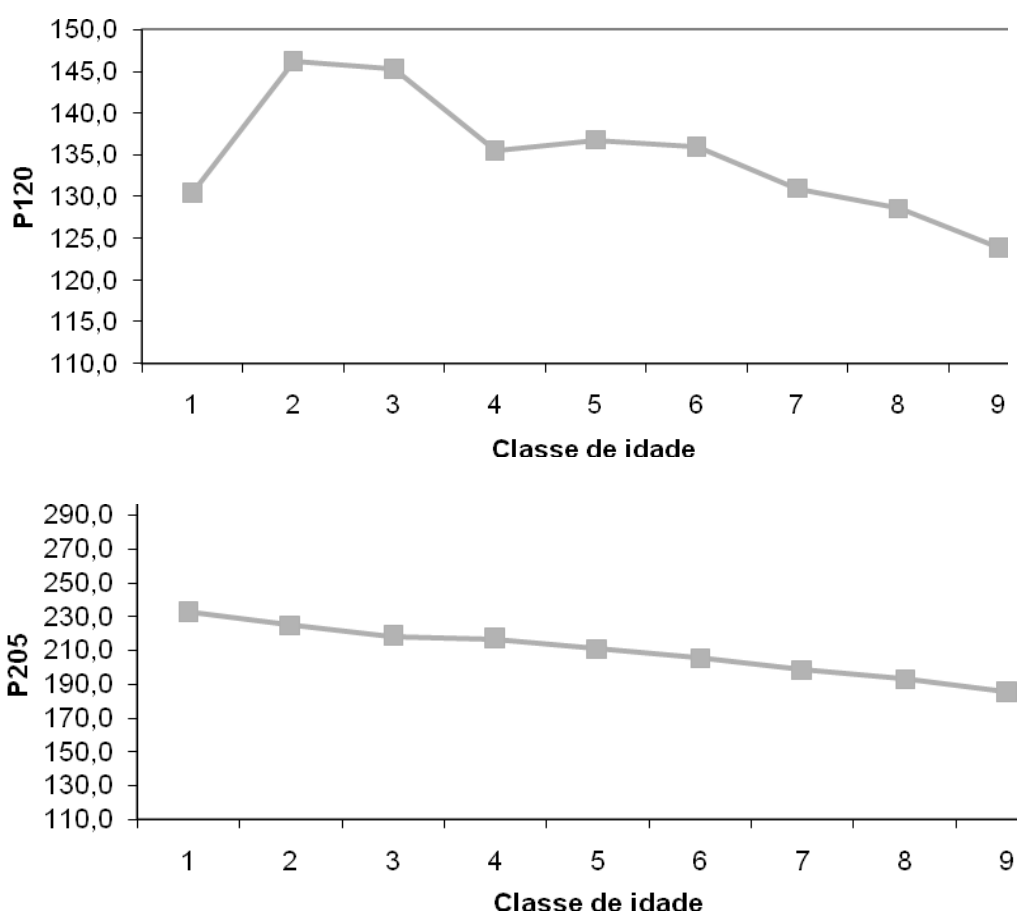

Figura 2. Médias ajustadas das características peso aos 120 (P120) e 205 (P205) dias de idade de acordo com a classe de idade da mensuração (CID) de bovinos cruzados Angus X Nelore

A interação entre CIMP e HTM foi importante e significativa para as duas características. $\mathrm{O}$ efeito demonstrou que atua de forma diferente conforme a composição racial da mãe e sua idade ao parto. Além disso, os resultados encontrados sugerem que ambos os efeitos relacionados com a mãe, idade ao parto e heterozigose materna, possibilitam maior ganho de peso dos bezerros e, consequentemente, maior produtividade (Figura 3).

$\mathrm{O}$ efeito da interação foi similar entre as características, apresentando mudanças pequenas entre as CIMP. As progênies de mães com HTM igual a 0,38 apresentaram os menores pesos independente da classe de idade da mãe ao parto, confirmando a importância de manter valores de heterozigose materna altos para obtenção de maior produtividade nos animais cruzados.

O modelo 2 apresentou menor valor de componente de variância residual para P120 e menor valor do $-2 \log (\mathrm{p})$ e do DIC para as duas características, sugerindo ser o modelo que apresenta melhores estimativas dos componentes de (co)variâncias (Tabela 5).

Valores das estimativas de herdabilidade a posteriori obtidas para os três modelos estudados apresentaram 
Rev. Bras. Saúde Prod. Anim., Salvador, v.16, n.2, p.278-289 abr./jun.., 2015 http://www.rbspa.ufba.br ISSN 15199940

valores próximos (Tabela 6). A estimativa de herdabilidade foi superior no modelo 2 para a característica P120. $\mathrm{O}$ mesmo modelo resultou em estimativa de herdabilidade menor para a característica P205 quando comparado

com os modelos 1 e 3 , entretanto o intervalo de credibilidade das estimativas de herdabilidade a posteriori para o P205 foi menor, sugerindo melhor ajustamento para esta característica.
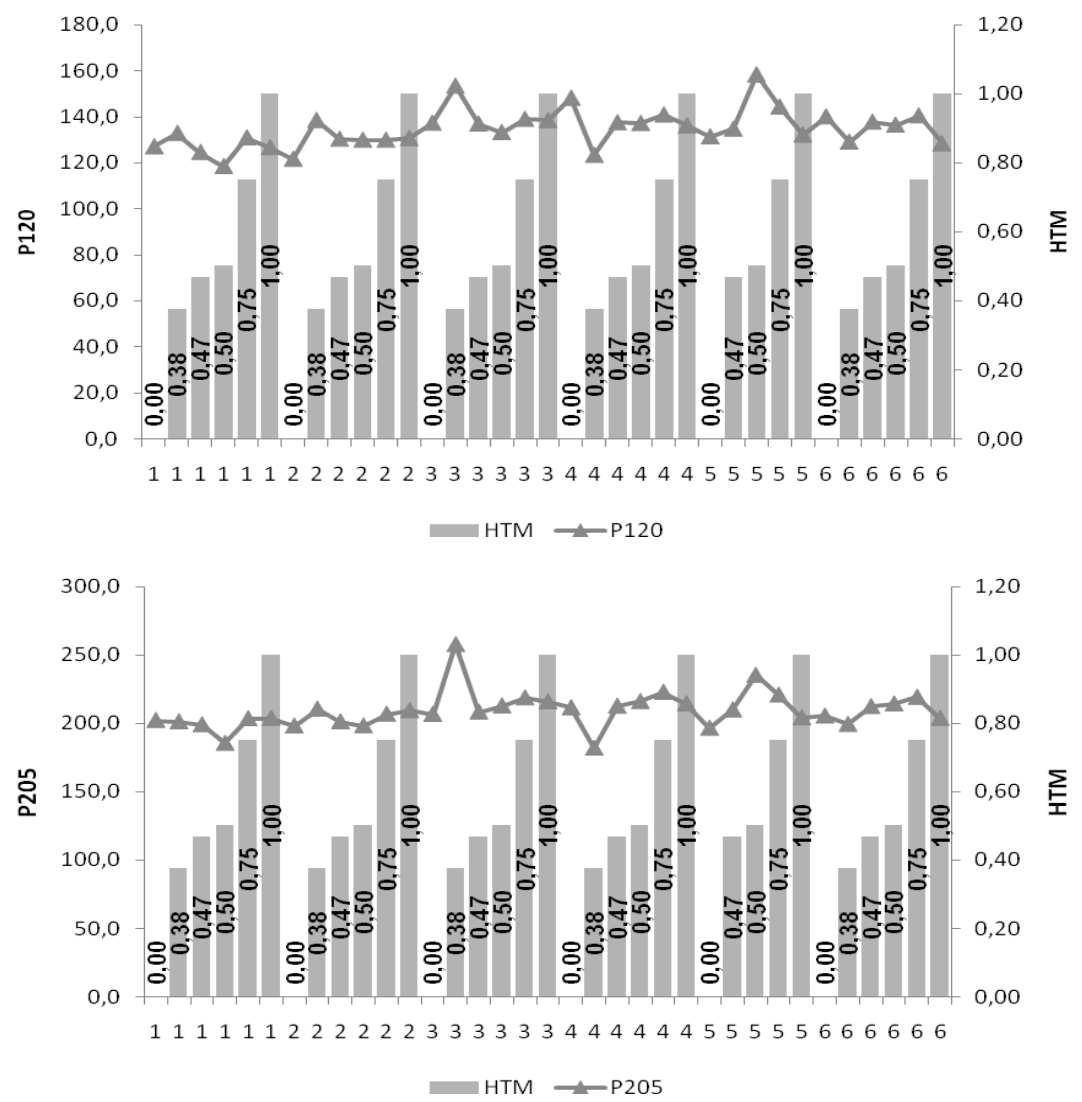

Figura 3. Médias ajustadas das características peso aos 120 (P120) e 205 (P205) dias de idade de acordo com a interação de classe de idade da mãe ao parto e heterozigose materna de bovinos cruzados Angus X Nelore

Tabela 5. Valores de consistência para os diferentes modelos aplicados nas análises bicaracterísticas das características peso aos 120 (P120) e 205 (P205) dias de idade em bovinos cruzados Angus x Nelore

\begin{tabular}{lcccc}
\hline Análise & Ve_P120 & Ve_P205 & $-2 \log (\mathrm{p})$ & DIC \\
\hline Modelo 1 & 173,18 & 393,89 & $127.350,705$ & $128.431,068$ \\
Modelo 2 & 161,87 & 388,52 & $120.839,154$ & $121.959,229$ \\
Modelo 3 & 168,70 & 385,69 & $120.842,313$ & $128.523,668$ \\
\hline
\end{tabular}

Ve_P120 e Ve_P205 representam a variância residual para peso aos 120 e 205 dias de idade, respectivamente. 
Rev. Bras. Saúde Prod. Anim., Salvador, v.16, n.2, p.278-289 abr./jun.., 2015 http://www.rbspa.ufba.br ISSN 15199940

Tabela 6. Estimativa de herdabilidade a posteriori para as características peso aos 120 (P120) e 205 (P205) dias de idade em bovinos cruzados Angus x Nelore

\begin{tabular}{|c|c|c|c|c|c|c|}
\hline Modelo & Variável & Média & Mediana & Moda & DP & IC_95 \\
\hline \multirow[t]{2}{*}{1} & $\mathrm{~h}^{2}{ }_{-} \mathrm{P} 120$ & 0,17 & 0,17 & 0,19 & 0,04 & 0,10 a 0,26 \\
\hline & $\mathrm{h}^{2}$ P205 & 0,21 & 0,21 & 0,17 & 0,04 & 0,14 a 0,29 \\
\hline \multirow[t]{2}{*}{2} & $\mathrm{~h}^{2}{ }_{-} \mathrm{P} 120$ & 0,20 & 0,20 & 0,17 & 0,04 & 0,12 a 0,29 \\
\hline & $\mathrm{h}^{2} \_\mathrm{P} 205$ & 0,20 & 0,20 & 0,17 & 0,04 & 0,13 a 0,27 \\
\hline \multirow{2}{*}{3} & $\mathrm{~h}^{2}$ P 120 & 0,19 & 0,19 & 0,18 & 0,05 & 0,10 a 0,29 \\
\hline & $\mathrm{h}^{2}{ }_{2} \mathrm{P} 205$ & 0,21 & 0,20 & 0,18 & 0,04 & 0,14 a 0,29 \\
\hline
\end{tabular}
respectivamente; DP = desvio padrão; IC_95 = intervalo de credibilidade a 95\%.

O processo de seleção para as características peso aos 120 dias de idade e peso aos 205 dias de idade pode resultar em ganhos genéticos quando utilizadas como critérios de seleção em programas de melhoramento animal dado os valores obtidos das estimativas de herdabilidade. Em bovinos da raça Nelore, Souza et al. (2011) encontraram estimativas de herdabilidade de 0,18 e 0,14 para pesos ajustados aos 120 e 205 dias de idade, respectivamente. Valor de 0,23 foi encontrado por Araujo Neto et al. (2011) para herdabilidade da característica peso ajustado aos 120 dias de idade, e estimativa de herdabilidade de 0,24 foi obtida para peso à desmama em bovinos da raça Nelore. Everling et al. (2001) encontraram valor de 0,23 para peso à desmama em rebanhos de animais Angus-Nelore. Os valores obtidos no estudo são pertinentes e apresentam similaridade com as estimativas de herdabilidade encontradas na literatura.

As comparações por meio da correlação de Pearson e Spearman dos valores genéticos de todos os animais constantes no pedigree e somente os touros apresentaram-se na Tabela 7. Os valores genéticos foram obtidos para cada um dos modelos com os componentes genéticos obtidos no modelo 2, considerado o modelo de melhor estimação.

Tabela 7. Correlações de Pearson (acima da diagonal) e Spearman (abaixo da diagonal) dos valores genéticos de todos os animais constantes no pedigree (Todos) e de somente os touros com mais de 100 progênies (Touros 100) para os três modelos utilizados para as características peso aos 120 (P120) e 205 (P205) dias de idade em bovinos cruzados Angus x Nelore

\begin{tabular}{lcccc}
\hline Item & & Modelo 1 & Modelo 2 & Modelo 3 \\
\hline \multirow{2}{*}{ Todos } & Modelo 1 & & 0,99 & 0,95 \\
& Modelo 2 & 0,99 & & 0,96 \\
& Modelo 3 & 0,94 & 0,95 & \\
\hline \multirow{2}{*}{ Touros 100 } & Modelo 1 & & 0,99 & 0,93 \\
& Modelo 2 & 0,99 & & 0,94 \\
& Modelo 3 & 0,91 & 0,93 & \\
\hline
\end{tabular}


Os valores apresentados na Tabela 7 mostram que os modelos 1 e 2 não apresentam alterações na classificação dos animais, assim o uso da interação entre a idade da vaca ao parto e heterozigose materna ou destes efeitos separadamente, bem como o uso de classes de idade na mensuração ou idade na mensuração como covariável linear, não interfere na classificação dos animais para a característica $\mathrm{P} 205$.

Resultados similares foram obtidos para a característica P120. Os modelos 1 e 2 , quando comparados com o modelo 3 , apresentaram correlações dos valores genéticos abaixo de 0,99, indicando alterações mínimas na classificação dos animais. É importante lembrar que no modelo 3, a principal diferença foi o não uso do efeito da data juliana de nascimento, sugerindo sua importância e portanto, deveria ser considerado nos modelos para obtenção dos valores genéticos para as características analisadas.

Os efeitos ambientais de classe de data juliana ao nascimento, classe de idade da mensuração e a interação da classe de idade da mãe ao parto e da heterozigose materna devem ser consideradas no momento da escolha dos reprodutores de modo a permitir maior precisão na avaliação genética e esquemas de seleção mais acurados.

\section{REFERÊNCIAS}

ARAUJO NETO, F.R.; LOBO, R.B.; MOTA, M.D.S.; OLIVEIRA, H.N. Genetic parameter estimates and response to selection for weight and testicular traits in Nelore cattle.

Genetics and Molecular Research, v.10, p.3127-3140, 2011.
BOCCHI, A.L.; ALBUQUERQUE, L.G. Efeito da idade da vaca e da data juliana de nascimento sobre o ganho médio diário de bezerros de corte no período pré-desmame. Revista Brasileira de Zootecnia, v.57, p.524-532, 2005.

EVERLING, D.M.; FERREIRA, G.B.B.; RORATO, P.R.N.; ROSO, V.M.; MARION, A.E.; FERNANDES, H.D. Estimativas de herdabilidade e correlação genética para características de crescimento na fase de pré-desmama e medidas de perímetro escrotal ao sobreano em bovinos Angus-Nelore.

Revista Brasileira de Zootecnia, v.30, p.2002-2008, 2001.

JORGE JUNIOR, J.; PITA, F.V.C.; FRIES, L.A.; ALBUQUERQUE, L.G. Influência de alguns fatores de ambiente sobre os escores de conformação, precocidade e musculatura à desmama em um rebanho da raça Nelore. Revista Brasileira de Zootecnia, v.30, p.16971703, 2001.

MISZTAL, I.; TSURUTA, S.; STRABEL, T.; AUVRAY, B.; DRUET, T.; LEE, D.H. BLUPF90 and related programs (BGF90). In: WORLD CONGRESS ON GENETICS APPLIED TO LIVESTOCK PRODUCTION, 7. 2002, Montpellier, France. Proceedings... Montpellier, France, 2002.

PAZ, C.C.P.; ALBUQUERQUE, L.G.; FRIES, L.A. Efeitos ambientais sobre ganho de peso no período do nascimento ao desmame em bovinos da raça Nelore.

Revista Brasileira de Zootecnia, v.28, p.55-64, 1999.

PEREIRA, A.M.V.S.; MUNIZ, C.A.S.D. Efeitos ambientais sobre características pré-desmama em bovinos da Raça Nelore Mocha. Semina: Ciências Agrárias, v.34, p.359-366, 2013. 
Rev. Bras. Saúde Prod. Anim., Salvador, v.16, n.2, p.278-289 abr./jun.., 2015 http://www.rbspa.ufba.br

SOUZA, J.C.; SILVA, L.O.C.;

GONDO, A.; FREITAS, J.A.;

MALHADO, C.H.M.; FERRAR

FILHO, P.B.; SERENO, J.R.B.;

WEABER, R.L.; LAMBERSON, W.R.

Parametros e tendências genéticas do

peso de bovinos criados a pasto no

Brasil. Archivos Zootecnia, v.60, p.457-465, 2011.

SPIEGELHALTER, D.J.; BEST, N.G.; CARLIN, B.P.; VAN DER LINDE, A.

Bayesian measures of model complexity and fit. Journal of the Royal

Statistical Society: Series B

(Statistical Methodology), v.64, p.583-639, 2002.

STATISTICAL ANALYSIS SYSTEM

- SAS. User's guide. Version 8.2. Cary:

SAS Institute, 2001. 943p.

Data de recebimento: $16 / 01 / 2014$

Data de aprovação: 20/03/2015 